\title{
Nonlinear Evolution of Anisotropic Cosmological Power
}

\author{
Shin'ichiro Ando and Marc Kamionkowski \\ California Institute of Technology, Mail Code 130-33, Pasadena, California 91125, USA
}

(Received 5 November 2007; published 21 February 2008)

\begin{abstract}
There has been growing interest in the possibility of testing more precisely the assumption of statistical isotropy of primordial density perturbations. If it is to be tested with galaxy surveys at distance scales $\lesssim 10 \mathrm{Mpc}$, then nonlinear evolution of anisotropic power must be understood. To this end, we calculate the angular dependence of the power spectrum to third order in perturbation theory for a primordial power spectrum with a quadrupole dependence on the wave vector direction. Our results suggest that primordial power anisotropies will be suppressed by $\lesssim 7 \%$ in the quasilinear regime. We also show that the skewness in the statistically anisotropic theory differs by no more than $1 \%$ from that in the isotropic theory.
\end{abstract}

PACS numbers: $98.80 .-\mathrm{k}$

It is commonly assumed that primordial density perturbations are statistically isotropic, and statistical isotropy is a prediction of most structure-formation theories. The notion of statistical isotropy can be quantified, though, by considering models in which it is broken, and a growing literature has discussed physical models that produce primordial perturbations that are not statistically isotropic [1].

The manifestations of departures from statistical isotropy can take on many forms, but one simple example [2] predicts a primordial power spectrum $P(\boldsymbol{k})$ with a quadrupole dependence on the direction $\hat{\boldsymbol{k}}$ of the wave vector $\boldsymbol{k}$,

$$
P(\boldsymbol{k})=A(k)\left[1+g_{*} \mathcal{P}_{2}\left(\mu_{k}\right)\right],
$$

where $\mathcal{P}_{2}(x)=\left(3 x^{2}-1\right) / 2$ is the second Legendre polynomial, $\mu_{k}=\hat{\boldsymbol{k}} \cdot \hat{\boldsymbol{e}}$, and $\hat{\boldsymbol{e}}$ is a preferred direction. (Note that our $g_{*}$ is 3/2 times that in Ref. [2].) Reference [3] constructed a minimum-variance estimator of cosmic microwave background for the power-anisotropy amplitude $g_{*}$ and showed that the Planck satellite will be sensitive to a value of $g_{*}$ as small as $\sim 0.02$, a number which can be estimated roughly by $\sim 10 N_{\text {pix }}^{-1 / 2}$, where $N_{\text {pix }}$ is the number of statistically independent pixels on the sky detected by Planck.

Similar arguments suggest that the sensitivity of a galaxy survey, like the Sloan Digital Sky Survey [4] or TwoDegree Field [5], should have a comparable sensitivity. One issue that will arise, though, in testing statistical isotropy of primordial perturbations is the quasilinear evolution of the power spectrum. The root-mean-square density-perturbation amplitude becomes of order unity at distance scales $\sim 10 h^{-1} \mathrm{Mpc}$, and so quasilinear evolution of the density field must be taken into account if the mass distribution measured on these scales in the Universe today is to be used to infer the primordial mass distribution.

In this Letter, we study the nonlinear evolution of density perturbations to see how nonlinearity affects statistical isotropy. Does nonlinear evolution amplify departures from statistical isotropy? Or possibly suppress them? To take the first steps to address this question, we calculate the power spectrum, to third order in perturbation theory, under the assumption that primordial perturbations have the form given in Eq. (1). The bottom line is that quasilinear evolution suppresses departures from statistical isotropy, but by only $\$ 7 \%$ compared with the linear theory. Thus, galaxy surveys in the linear or quasilinear regime will still be useful diagnostics for departures from statistical isotropy. We also calculate skewness, finding that the quadrupole power anisotropy changes it by no more than $1 \%$.

To proceed, we use third-order perturbation theory to determine whether a primordial power anisotropy is amplified or suppressed in the quasilinear regime. The power spectrum $P(\boldsymbol{k}, z)$ is defined by the ensemble average of the two-point correlation of the Fourier transform $\hat{\delta}(\boldsymbol{k}, z)$ of the density perturbation through

$$
\left\langle\hat{\delta}\left(\boldsymbol{k}_{1}, z\right) \hat{\delta}\left(\boldsymbol{k}_{2}, z\right)\right\rangle=(2 \pi)^{3} \delta_{D}\left(\boldsymbol{k}_{1}+\boldsymbol{k}_{2}\right) P\left(\boldsymbol{k}_{1}, z\right),
$$

where $\delta_{D}$ is the Dirac delta function. The density perturbations can be expanded in terms of the linear-theory density-perturbation amplitude, which has a redshift dependence proportional to the linear growth factor $D(z)$,

$$
\hat{\delta}(\boldsymbol{k}, z)=\sum_{n=1}^{\infty} \delta_{n}(\boldsymbol{k}) D^{n}(z)
$$

In the linear regime, each Fourier mode grows at the same rate, and so the linear-theory power spectrum $P_{\operatorname{lin}}(\boldsymbol{k})$, corresponding to the linear density perturbation $\delta_{1}(\boldsymbol{k})$, evolves in such a way that the quadrupole dependence of the primordial power spectrum [Eq. (1)] is preserved. However, the nonlinear power spectrum will have different anisotropy structure, and we shall investigate it following the prescription developed in Refs. [6-8].

Reference [6] showed that solution of the nonrelativistic fluid equations (i.e., continuity, Euler, and Poisson equations) allows one to write the $n$ th-order densityperturbation amplitude $\delta_{n}$ in terms of the linear perturbation $\delta_{1}$ through 


$$
\begin{aligned}
\delta_{n}(\boldsymbol{k})= & \int \frac{d \boldsymbol{q}_{1}}{(2 \pi)^{3}} \cdots \int \frac{d \boldsymbol{q}_{n}}{(2 \pi)^{3}} \delta_{D}\left(\boldsymbol{q}_{1}+\cdots+\boldsymbol{q}_{n}-\boldsymbol{k}\right) \\
& \times(2 \pi)^{3} F_{n}\left(\boldsymbol{q}_{1}, \cdots, \boldsymbol{q}_{n}\right) \delta_{1}\left(\boldsymbol{q}_{1}\right) \cdots \delta_{1}\left(\boldsymbol{q}_{n}\right) .
\end{aligned}
$$

For any $n$, the function $F_{n}$ can be obtained by recursive relations given in Ref. [6]. In particular, the expressions for
$F_{2}$ and $F_{3}$ that are directly relevant for our purpose are explicitly given by Eqs. (A2) and (A3) of Ref. [6]. (Strictly speaking, $F_{2}$ in Ref. [6] is for an Einstein-de Sitter universe, but Ref. [9] shows that its form in a $\Lambda$ CDM model with $\Omega_{m} \simeq 0.3$ differs by less than $1 \%$; the same should be true for $F_{3}$.) As all odd moments of $\delta_{1}(\boldsymbol{k})$ vanish, the power spectrum to third order is given as

$$
(2 \pi)^{3} \delta_{D}\left(\boldsymbol{k}_{1}+\boldsymbol{k}_{2}\right) P^{(2)}\left(\boldsymbol{k}_{1}, z\right)=D^{2}(z)\left\langle\delta_{1}\left(\boldsymbol{k}_{1}\right) \delta_{1}\left(\boldsymbol{k}_{2}\right)\right\rangle+D^{4}(z)\left[\left\langle\delta_{2}\left(\boldsymbol{k}_{1}\right) \delta_{2}\left(\boldsymbol{k}_{2}\right)\right\rangle+\left\langle\delta_{1}\left(\boldsymbol{k}_{1}\right) \delta_{3}\left(\boldsymbol{k}_{2}\right)\right\rangle+\left\langle\delta_{3}\left(\boldsymbol{k}_{1}\right) \delta_{1}\left(\boldsymbol{k}_{2}\right)\right\rangle\right]
$$

The second term, which evolves as $D^{4}(z)$, is the next-toleading term evaluated to the third order of density perturbation $\delta_{3}$, while the first term is the linear part. We further define a quantity $P_{m n}$ as

$$
\left\langle\delta_{m}\left(\boldsymbol{k}_{1}\right) \delta_{n}\left(\boldsymbol{k}_{2}\right)\right\rangle=(2 \pi)^{3} \delta_{D}\left(\boldsymbol{k}_{1}+\boldsymbol{k}_{2}\right) P_{m n}\left(\boldsymbol{k}_{1}\right),
$$

and with this definition, Eq. (5) is rewritten as

$$
P^{(2)}(\boldsymbol{k}, z)=D^{2}(z) P_{11}(\boldsymbol{k})+D^{4}(z)\left[P_{22}(\boldsymbol{k})+2 P_{13}(\boldsymbol{k})\right] .
$$

We then need to write $P_{22}$ and $P_{13}$ in terms of the linear spectrum $P_{\text {lin }}=P_{11}$. This procedure is straightforward, and the results are very similar to those given in Ref. [8]:

$$
\begin{gathered}
P_{22}(\boldsymbol{k})=2 \int \frac{d \boldsymbol{q}}{(2 \pi)^{3}}\left[F_{2}^{(s)}(\boldsymbol{q}, \boldsymbol{k}-\boldsymbol{q})\right]^{2} P_{\operatorname{lin}}(\boldsymbol{q}) P_{\operatorname{lin}}(\boldsymbol{k}-\boldsymbol{q}), \\
P_{13}(\boldsymbol{k})=3 P_{\operatorname{lin}}(\boldsymbol{k}) \int \frac{d \boldsymbol{q}}{(2 \pi)^{3}} F_{3}^{(s)}(\boldsymbol{q},-\boldsymbol{q}, \boldsymbol{k}) P_{\operatorname{lin}}(\boldsymbol{q}),
\end{gathered}
$$

where the symmetrized function $F_{n}^{(s)}$ is obtained by summing $n$ ! permutations of $F_{n}$ over its $n$ arguments and dividing by $n$ !. The only difference from the expressions in Ref. [8] is that the linear power spectrum now depends on both magnitude $k$ and direction $\hat{\boldsymbol{k}}$ of wave vector $\boldsymbol{k}$.

In order to illuminate the anisotropy structure, we expand Eqs. (8) and (9) with Legendre polynomials. The most general power spectrum today $(D=1)$ can be expanded in Legendre polynomials $\mathcal{P}_{n}(x)$ as

$$
P(\boldsymbol{k})=P_{\text {lin }}\left(k, \mu_{k}\right)+\sum_{m, n=0}^{\infty} g_{*}^{m} \mathcal{P}_{n}\left(\mu_{k}\right) B_{m n}(k),
$$

where the sum on $n$ is restricted to even positive integers. To the next-to-leading order, the expansion can be written,

$$
\begin{aligned}
P^{(2)}(\boldsymbol{k})= & A_{\text {lin }}(k)+B_{00}(k)+g_{*}^{2} B_{20}(k)+\left\{g _ { * } \left[A_{\operatorname{lin}}(k)\right.\right. \\
& \left.\left.+B_{12}(k)\right]+g_{*}^{2} B_{22}(k)\right\} \mathcal{P}_{2}(k)+g_{*}^{2} B_{24}(k) \mathcal{P}_{4}(k),
\end{aligned}
$$

where $A_{\text {lin }}$, defined by Eq. (1), is the isotropic linear power spectrum. Expressions for the expansion coefficients $B_{m n}(k)$ are given at the end of the Letter. We then rewrite Eq. (11) as

$$
P^{(2)}(\boldsymbol{k})=A^{(2)}(k)\left[1+g_{2}^{(2)}(k) \mathcal{P}_{2}\left(\mu_{k}\right)+g_{4}^{(2)}(k) \mathcal{P}_{4}\left(\mu_{k}\right)\right],
$$

$$
\begin{gathered}
g_{2}^{(2)}(k)=g_{*} c_{1}(k)+g_{*}^{2} c_{2}(k), \\
g_{4}^{(2)}(k)=g_{*}^{2} c_{3}(k),
\end{gathered}
$$

with the following definitions of $A^{(2)}, c_{1}, c_{2}$, and $c_{3}$ :

$$
\begin{array}{r}
A^{(2)}(k)=A_{\operatorname{lin}}(k)+B_{00}(k)+g_{*}^{2} B_{20}(k), \\
A^{(2)}(k) c_{i}(k)= \begin{cases}A_{\operatorname{lin}}(k)+B_{12}(k) & {[i=1]} \\
B_{22}(k) & {[i=2] .} \\
B_{24}(k) & {[i=3]}\end{cases}
\end{array}
$$

We note that the primordial quadrupole anisotropy also affects the isotropic part of the next-to-leading order power spectrum, $A^{(2)}(k)$. While this is interesting, that correction is expected to be very small, being suppressed by $g_{*}^{2}$. Thus, hereafter we neglect this term; keeping it only gives correction of the order of $g_{*}^{3}$ and $g_{*}^{4}$ to $g_{2}^{(2)}$ and $g_{4}^{(2)}$, respectively.

For the linear power spectrum $A_{\text {lin }}(k)$, we use the fitting formula for the transfer function given in Ref. [10] with current values for the relevant cosmological parameters [11].

If $g_{*} \ll 1$, we may neglect all the terms proportional to $g_{*}^{2}$, and thus $c_{1}(k)$ is the only quantity relevant for anisotropy structure. As linear theory simply gives $c_{1}(k)=1$, the quantity $c_{1}(k)-1$ represents enhancement of the quadrupole anisotropy due to quasilinear evolution. In Fig. 1, we plot $c_{1}(k)-1$. This figure shows that at large scales $k \lessgtr$ $10^{-2} \mathrm{Mpc}^{-1}$, the nonlinear effect is subdominant and thus anisotropic structure is the same as that for the linear theory: $c_{1}(k) \approx 1$. The quadrupole anisotropy then decreases at smaller scales and it reaches minimum $\left(c_{1}-1 \simeq\right.$ $-0.07)$ in the quasilinear regime, $k \sim 0.1 \mathrm{Mpc}^{-1}$.

Third-order perturbation theory becomes less accurate for even higher wave numbers, $k \gtrsim 0.1 \mathrm{Mpc}^{-1}$, at $z=0$. However, it remains very accurate at such (comoving) scales in the high-redshift universe, $z \geq 1$ [12]. For general redshifts $z$, the anisotropic power spectrum is given by Eqs. (12)-(16) with replacements $A_{\operatorname{lin}} \rightarrow D^{2}(z) A_{\operatorname{lin}}$ and $B_{m n} \rightarrow D^{4}(z) B_{m n}$. The enhancement of anisotropy is then given by $c_{1}(k, z)-1$, which is well approximated by $\left[c_{1}(k)-1\right] D^{2}(z)$ in the quasilinear regime where $A_{\text {lin }}>$ $\left|B_{m n}\right|$. It is -0.03 at $k=0.1 \mathrm{Mpc}^{-1}$ and $z=1$; the suppression is weaker. One can also see amplified anisotropy for $k \geq 0.3 \mathrm{Mpc}^{-1}$ at redshifts when the third-order approach is still valid at such scales.

The corrections on the order of $g_{*}^{2}$ are represented by $c_{2}(k)$ and $c_{3}(k)$, both of which are also shown in Fig. 1 . 


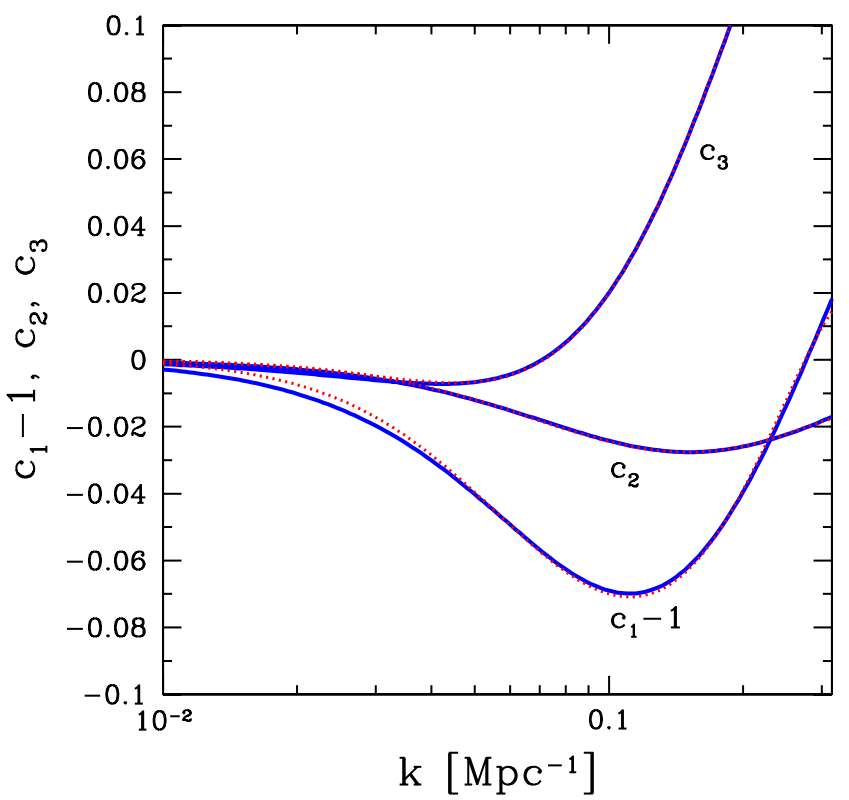

FIG. 1 (color online). Coefficients of anisotropic terms in the third-order power spectrum, $c_{1}(k)-1, c_{2}(k)$, and $c_{3}(k)$. The definitions are given in Eqs. (12)-(14). Solid curves are the result of numerical integration, while dotted curves are the fitting functions (17)-(19).

Besides it is suppressed by additional $g_{*}, c_{2}(k)$ is intrinsically smaller than $c_{1}-1$, thus giving only a minor correction. While possessing different anisotropy structure, the higher multipole term proportional to $c_{3}(k)$ would also be small in the quasilinear regime.

The results of our computations can be approximated by

$$
\begin{aligned}
c_{1}(k)= & 1-0.463 \Delta^{2}(k)+0.886 \Delta^{4}(k)-0.407 \Delta^{6}(k), \\
c_{2}(k)= & -0.133 \Delta^{2}(k)+0.195 \Delta^{4}(k)-0.079 \Delta^{6}(k), \\
c_{3}(k)= & -0.163 \Delta^{2}(k)+1.062 \Delta^{4}(k)-1.082 \Delta^{6}(k) \\
& +0.373 \Delta^{8}(k),
\end{aligned}
$$

in terms of the linear-theory density-perturbation amplitude at wave number $k, \Delta^{2}(k)=\left(k^{3} / 2 \pi^{2}\right) A_{\operatorname{lin}}(k)$. These fitting functions are plotted as dotted curves in Fig. 1. They were obtained explicitly for the current best-fit cosmological parameters [11], but when written in terms of $\Delta^{2}(k)$, should also be accurate for other cosmological parameters. Strictly speaking, the validity of third-order perturbation theory breaks down when the terms higher order in $\Delta^{2}(k)$ become important; in this case, one will need to go to fourth order or even higher-order corrections to obtain the evolved power spectrum.

We now compute the skewness $S_{3} \equiv\left\langle\delta^{3}\right\rangle /\left\langle\delta^{2}\right\rangle^{2}$ from the anisotropic primordial power spectrum, to second order in perturbation theory. The standard result, for an Einsteinde Sitter universe, is $S_{3}=34 / 7[13,14]$, and the result for a $\Lambda \mathrm{CDM}$ universe differs by less than $1 \%$. The three-point correlation function at zero lag is a Fourier transform of $\left\langle\hat{\delta}\left(\boldsymbol{k}_{1}, z\right) \hat{\delta}\left(\boldsymbol{k}_{2}, z\right) \hat{\delta}\left(\boldsymbol{k}_{3}, z\right)\right\rangle$, and thus the leading contribution comes from $D^{4}(z)\left\langle\delta_{1}\left(\boldsymbol{k}_{1}\right) \delta_{1}\left(\boldsymbol{k}_{2}\right) \delta_{2}\left(\boldsymbol{k}_{3}\right)\right\rangle$, etc. Rewriting $\delta_{2}$ in terms of the linear fluctuation $\delta_{1}$ and the integration kernel $F_{2}$, and using the definition of linear power spectrum, we obtain

$$
\begin{aligned}
\left\langle\delta^{3}(z)\right\rangle= & 6 D^{4}(z) \int \frac{d \boldsymbol{k}_{1}}{(2 \pi)^{3}} \int \frac{d \boldsymbol{k}_{2}}{(2 \pi)^{3}} F_{2}^{(s)}\left(\boldsymbol{k}_{1}, \boldsymbol{k}_{2}\right) \\
& \times P_{\operatorname{lin}}\left(\boldsymbol{k}_{1}\right) P_{\operatorname{lin}}\left(\boldsymbol{k}_{2}\right) .
\end{aligned}
$$

After integrating over the directions of wave vectors, $\hat{\boldsymbol{k}}_{1}$ and $\hat{\boldsymbol{k}}_{2}$, we find

$$
\left\langle\delta^{3}(z)\right\rangle=D^{4}(z)\left(\frac{34}{7}+\frac{8 g_{*}^{2}}{175}\right)\left[\int \frac{d k k^{2}}{2 \pi^{2}} A_{\operatorname{lin}}(k)\right]^{2} .
$$

On the other hand, the variance $\left\langle\delta^{2}\right\rangle$ can be computed in a similar manner, and to the same order, we obtain

$$
\left\langle\delta^{2}(z)\right\rangle=D^{2}(z) \int \frac{d \boldsymbol{k}}{(2 \pi)^{3}} P_{\operatorname{lin}}(\boldsymbol{k})=D^{2}(z) \int \frac{d k k^{2}}{2 \pi^{2}} A_{\operatorname{lin}}(k) .
$$

Therefore, we find the skewness to be

$$
S_{3}=\frac{\left\langle\delta^{3}(z)\right\rangle}{\left\langle\delta^{2}(z)\right\rangle^{2}}=\frac{34}{7}+\frac{8 g_{*}^{2}}{175} .
$$

The requirement that the power spectrum be positive definite restricts the value $0<g_{*}<1$, and so the largest possible deviation from the isotropic value of $34 / 7$ is less than $1 \%$. Thus, the skewness will not be an effective discriminant for anisotropic power.

To conclude, we used third-order perturbation theory to calculate the quasilinear evolution of a primordial power spectrum. Our results show that nonlinear evolution suppresses the primordial anisotropy. The suppression is $\sim 7 \%$ at $k=0.1 \mathrm{Mpc}^{-1}$ and $z=0$ compared with the linear theory. This must be taken into account when interpreting the result of searches in the quasilinear regime for primordial anisotropy, and it indicates that nonlinear scales are still valuable as tests for the isotropy of primordial power, since the suppression is weak. We also found that the standard prediction for the skewness is little changed if the primordial power spectrum has a quadrupole anisotropy.

Our perturbative results seem to indicate that a quadrupole anisotropy is enhanced at smaller scales, but this enhancement cannot be trusted at low redshifts, as it occurs at scales where our perturbative approach breaks down. It seems counterintuitive to think that growth of perturbations in the highly nonlinear regime can act to enhance the primordial anisotropy, but it will require an $\mathrm{N}$-body simulation to be sure. Likewise, we imagine that departures from statistical isotropy that are higher order in angle (octupole, etc.) will also be suppressed by quasilinear evolution, but we leave that calculation for future work.

Before closing, we provide explicit expressions for the quantities in Eq. (10). For the numerical evaluation of Eqs. (8) and (9), we shall choose $\boldsymbol{k}$ along the $z$-direction, 
$\hat{\boldsymbol{k}}=(0,0,1)$, and the preferred direction $\hat{\boldsymbol{e}}$ in the $x$ - $z$ plane,

$\hat{\boldsymbol{e}}=\left(\sqrt{1-\mu_{k}^{2}}, 0, \mu_{k}\right)$. We then use the spherical coordinate for $\boldsymbol{q}=q\left(\sqrt{1-\mu^{2}} \cos \phi, \sqrt{1-\mu^{2}} \sin \phi, \mu\right)$, and $d \boldsymbol{q}=q^{2} d q d \mu d \boldsymbol{\phi}$. With this notation, $B_{m n}$ in Eq. (10) are given as

$$
\begin{gathered}
B_{m n}=S_{m n}+2 T_{m n}, \\
S_{m n}=\frac{k^{4}}{\pi^{2}} \int_{0}^{\infty} d q \int_{-1}^{1} d \mu \frac{\left[7 k \mu+q\left(3-10 \mu^{2}\right)\right]^{2}}{\left(k^{2}+q^{2}-2 k q \mu\right)^{3}} \\
\times I_{m n} A_{\operatorname{lin}}(q) A_{\operatorname{lin}}\left(\sqrt{k^{2}+q^{2}-2 k q \mu}\right), \\
T_{m n}=\frac{k^{2} A_{\operatorname{lin}}(k)}{\pi^{2}} \int_{0}^{\infty} d q A_{\operatorname{lin}}(q)\left[\mathcal{J}_{m n}\right. \\
\left.+\mathcal{K}_{m n}\left(q^{2}-k^{2}\right)^{3} \ln \left(\frac{k+q}{|k-q|}\right)\right],
\end{gathered}
$$

where $S_{m n}$ and $T_{m n}$ correspond to $P_{22}$ and $P_{13}$, respectively. Explicit forms of $I_{m n}, \mathcal{J}_{m n}$, and $\mathcal{K}_{m n}$ are

$$
\begin{gathered}
I_{00}=\frac{k^{2}+q^{2}-2 k q \mu}{392} \\
I_{12}=\frac{2 q^{2}\left(3 \mu^{2}-1\right)+\left(k^{2}-2 k q \mu\right)\left(3 \mu^{2}+1\right)}{784}, \\
I_{20}=\frac{2 q^{2}-4 k q \mu+k^{2}\left(3 \mu^{2}-1\right)}{3920}, \\
I_{22}=\frac{\left(k^{2}+q^{2}\right)\left(3 \mu^{2}-1\right)-k q \mu\left(3 \mu^{2}+1\right)}{2744}, \\
-\frac{9}{54880}\left[4 k^{2}\left(3 \mu^{2}-1\right)+q^{2}\left(35 \mu^{4}-30 \mu^{2}+3\right)\right. \\
\mathcal{J}_{00}=\frac{1}{1008}\left(6 \frac{k^{2}}{q^{2}}-79+50 \frac{q^{2}}{k^{2}}-21 \frac{q^{4}}{k^{4}}\right) \\
\mathcal{J}_{12}=\frac{1}{40320}\left(90 \frac{k^{4}}{q^{4}}+375 \frac{k^{2}}{q^{2}}-5006+1732 \frac{q^{2}}{k^{2}}\right. \\
\left.-300 \frac{q^{4}}{k^{4}}-315 \frac{q^{6}}{k^{6}}\right), \\
\mathcal{J}_{20}=\frac{7}{10} \mathcal{J}_{22}=\frac{7}{18} \mathcal{J}_{24} \\
\left.+540 \frac{\mathcal{K}_{00}}{k^{4}}-315 \frac{q^{6}}{k^{6}}\right) \\
600 \frac{k^{4}}{q^{4}}+135 \frac{k^{2}}{q^{2}}-1846-268 \frac{q^{2}}{k^{2}}
\end{gathered}
$$

$$
\begin{aligned}
\mathcal{K}_{12} & =\frac{6 k^{6}+41 k^{4} q^{2}+76 k^{2} q^{4}+21 q^{6}}{5376 k^{7} q^{5}}, \\
\mathcal{K}_{20} & =\frac{7}{10} \mathcal{K}_{22}=\frac{7}{18} \mathcal{K}_{24} \\
& =\frac{6 k^{6}+25 k^{4} q^{2}+20 k^{2} q^{4}+21 q^{6}}{26880 k^{7} q^{5}},
\end{aligned}
$$

and all the other components vanish. Note that $T_{00}$ is the same as Eq. (19) of Ref. [8].

M. K. acknowledges the hospitality of the Aspen Center for Physics, where part of this work was completed. This work was supported by the Sherman Fairchild Foundation (S. A.), DoE No. DE-FG03-92-ER40701, NASA No. NNG05GF69G, and the Gordon and Betty Moore Foundation (M. K.).

[1] C. Gordon, W. Hu, D. Huterer, and T. Crawford, Phys. Rev. D 72, 103002 (2005); J. G. Cresswell, A. R. Liddle, P. Mukherjee, and A. Riazuelo, Phys. Rev. D 73, 041302 (2006); S.H.S. Alexander, arXiv:hep-th/0601034; A. Berera, R. V. Buniy, and T. W. Kephart, J. Cosmol. Astropart. Phys. 10 (2004) 016; R. V. Buniy, A. Berera, and T. W. Kephart, Phys. Rev. D 73, 063529 (2006); G. V. Chibisov and Yu. V. Shtanov, Int. J. Mod. Phys. A 5, 2625 (1990); G. V. Chibisov and Yu. V. Shtanov, Sov. Phys. JETP 69, 17 (1989) [Zh. Eksp. Teor. Fiz. 96, 32 (1989)]; A.E. Gumrukcuoglu, C.R. Contaldi, and M. Peloso, J. Cosmol. Astropart. Phys. 11 (2007) 005; C. ArmendarizPicon, J. Cosmol. Astropart. Phys. 09 (2007) 014; J. F. Donoghue, K. Dutta, and A. Ross, arXiv:astro-ph/ 0703455; R. A. Battye and A. Moss, Phys. Rev. D 74, 041301 (2006); C.G. Bohmer and D.F. Mota, arXiv:0710.2003; T. S. Pereira, C. Pitrou, and J. P. Uzan, J. Cosmol. Astropart. Phys. 09 (2007) 006.

[2] L. Ackerman, S. M. Carroll, and M. B. Wise, Phys. Rev. D 75, 083502 (2007).

[3] A. R. Pullen and M. Kamionkowski, Phys. Rev. D 76, 103529 (2007).

[4] http://www.sdss.org.

[5] http://www.aao.gov.au/2df.

[6] M.H. Goroff, B. Grinstein, S. J. Rey, and M. B. Wise, Astrophys. J. 311, 6 (1986).

[7] N. Makino, M. Sasaki, and Y. Suto, Phys. Rev. D 46, 585 (1992).

[8] B. Jain and E. Bertschinger, Astrophys. J. 431, 495 (1994).

[9] M. Kamionkowski and A. Buchalter, Astrophys. J. 514, 7 (1999).

[10] D. J. Eisenstein and W. Hu, Astrophys. J. 511, 5 (1999).

[11] D. N. Spergel et al., Astrophys. J. Suppl. Ser. 170, 377 (2007).

[12] D. Jeong and E. Komatsu, Astrophys. J. 651, 619 (2006).

[13] P. J. E. Peebles, The Large-Scale Structure of the Universe (Princeton University, Princeton, 1980).

[14] J. N. Fry, Astrophys. J. 279, 499 (1984). 